\title{
What is the clinical effectiveness and cost-effectiveness of using digital health technologies to improve treatment adherence and outcomes in patients with tuberculosis?
}

\author{
Authors: Kartik Kumar ${ }^{A}$ and Saira Ghafur ${ }^{B}$
}

\section{Introduction}

Non-adherence to tuberculosis (TB) therapy increases the risk of treatment failure, leading to poor clinical outcomes and a significant financial burden to healthcare services. Digital health technologies may offer innovative solutions to this, but studies evaluating their utility remain sparse. To address this research gap, a systematic review was undertaken to evaluate the existing evidence for the clinical effectiveness and cost-effectiveness of using digital health technologies to improve TB treatment adherence and clinical outcomes.

\section{Materials and methods}

Articles were retrieved by systematically searching MEDLINE, EMBASE, Web of Science, Scopus, CENTRAL, ClinicalTrials.gov, World Health Organization (WHO) Clinical Trials Registry Platform, WHO publications and the grey literature. Technologies under consideration were short message service (SMS), smartphone applications, medication monitors, video observed therapy (VOT), ingestible sensors with wirelessly observed therapy (WOT) and social media platforms. Studies were included if they were randomised controlled trials (RCTs), observational studies with controls or economic studies. Primary outcome measures were treatment adherence, cure, treatment completion, treatment failure, loss to follow-up and treatment success, as defined by WHO. ${ }^{1}$ A further outcome measure of interest was any related cost or cost-effectiveness data.

\section{Results and discussion}

4,044 records were initially identified. After removal of duplicate records and articles unrelated to either TB or digital health technologies, 111 records remained. Of these, 16 articles were eligible for inclusion in the qualitative analysis.

\section{SMS}

Compared to directly observed therapy (DOT), one-way SMS improved TB treatment completion ${ }^{2}$ but did not improve treatment

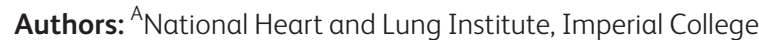
London, London, UK; Imperial College Healthcare NHS Trust, London, UK; ${ }^{\mathrm{B}}$ Centre for Health Policy, Institute of Global Health Innovation, Imperial College London, London, UK success or cure rates. ${ }^{3}$ The remaining one-way SMS studies and all the two-way SMS studies did not demonstrate significant improvements in adherence or outcomes. ${ }^{4-8}$ An economic study on SMS use in TB care demonstrated an incremental costeffectiveness ratio of 350 'international dollars' per disability adjusted life year. ${ }^{9}$

\section{Smartphone applications}

The one study on smartphone applications did not demonstrate an improvement in TB treatment outcomes. ${ }^{10}$ No evidence was available regarding cost-effectiveness.

\section{Medication monitors}

Medication monitors may improve TB cure rates compared to $\mathrm{DOT}^{11}$ and, when used alone or in conjunction with SMS, they may decrease treatment non-adherence rates, but they did not affect treatment failure rates, death or loss to follow-up when compared to standard self-administered treatment or DOT. ${ }^{8}$ A cost minimisation analysis projected lower costs per patient when using medication monitors compared to DOT. ${ }^{11}$

VOT

VOT did not improve TB treatment completion rates compared to DOT. ${ }^{12,13}$ Economic studies suggested that VOT may be associated with cost savings. ${ }^{13-16}$

\section{Ingestible sensors with WOT}

No eligible studies were identified regarding the clinical effectiveness of WOT in TB treatment. One economic modelling study suggested that using WOT may lower costs compared to DOT. ${ }^{17}$

\section{Social media platforms}

No eligible studies were identified regarding the clinical effectiveness or cost-effectiveness of using social media platforms in the management of TB.

\section{Conclusion}

There is currently insufficient evidence available on the clinical effectiveness and cost-effectiveness of using these digital health 
technologies to improve TB treatment adherence and outcomes. These findings will be of relevance to health policymakers who determine how best to invest in resources for TB control and to healthcare providers seeking to establish which technologies may be of value at a local level. The paucity in evidence means that, at present, policymakers cannot make definitive evidence-based decisions regarding wider implementation of these technologies. Further robust studies are needed, particularly in regions with the highest TB burden.

\section{References}

1 World Health Organization. Definitions and reporting framework for tuberculosis. Geneva: WHO, 2014. http://apps.who.int/iris/bitstream/handle/10665/79199/9789241505345_eng.pdf;jsessionid = 6F1FE251DDCFC1E6E9828EABC57E8337? sequence $=1$ [Accessed 15 December 2018].

2 Fang XH, Guan SY, Tang L et al. Effect of short message service on management of pulmonary tuberculosis patients in Anhui Province, China: A prospective, randomized, controlled study. Med Sci Monit 2017:23:2465-9.

3 Bediang G, Stoll B, Elia N, Abena J, Geissbuhler A. Short message service reminders to improve adherence and cure of tuberculosis patients in Cameroon (TB-SMS Cameroon): a randomised controlled trial. BMC Public Health 2018;18:83.

4 Johnston JC, van der Kop ML, Smillie K et al. The effect of text messaging on latent tuberculosis treatment adherence: a randomised controlled trial. Eur Respir ] 2018:51:1701488.

5 Farooqi RJ, Ashraf S, Zaman M. The role of mobile SMS-reminders in improving drugs compliance in patients receiving anti-TB treatment from DOTS program. Journal of Postgraduate Medical Institute 2017;31:156-62.

6 Mohammed S, Glennerster R, Khan AJ. Impact of a daily SMS medication reminder system on tuberculosis treatment outcomes: A randomized controlled trial. PLoS One 2016;11:e0162944.
7 Iribarren S, Beck S, Pearce PF et al. TextTB: A mixed method pilot study evaluating acceptance, feasibility, and exploring initial efficacy of a text messaging intervention to support TB treatment adherence. Tuberc Res Treat 2013;2013:349394.

8 Liu X, Lewis J], Zhang $\mathrm{H}$ et al. Effectiveness of electronic reminders to improve medication adherence in tuberculosis patients: a cluster-randomised trial. PLoS Med 2015;12:e1001876.

9 Hunchangsith P, Barendregt J], Vos T, Bertram M. Costeffectiveness of various tuberculosis control strategies in Thailand. Value Health 2012;15(Suppl 1):S50-5.

10 Promthong K, Kittivoravitkul P, Oer-Areemitr N, Wattanathum A. Tuberculosis treatment with smartphone medical application reminders a randomised control study. Respirology 2016;21(Suppl 3):96.

11 Broomhead S, Mars M. Retrospective return on investment analysis of an electronic treatment adherence device piloted in the Northern Cape Province. Telemed J E Health 2012;18;24-31.

12 Chuck C, Robinson E, Macaraig M, Alexander M, Burzynski J. Enhancing management of tuberculosis treatment with video directly observed therapy in New York City. Int J Tuberc Lung Dis 2016;20:588-93.

13 Wade VA, Karnon J, Eliott JA, Hiller JE. Home videophones improve direct observation in tuberculosis treatment: a mixed methods evaluation. PLoS One 2012; 7:e50155.

14 Holzman SB, Zenilman A, Shah M. Advancing patient-centered care in tuberculosis management: a mixed-methods appraisal of video directly observed therapy. Open Forum Infect Dis 2018;5:ofy046.

15 Mirsaeidi M, Farshidpour M, Banks-Tripp D et al. Video directly observed therapy for treatment of tuberculosis is patient-oriented and cost-effective. Eur Respir ] 2015;46:871-4.

16 Krueger K, Ruby D, Cooley P et al. Videophone utilization as an alternative to directly observed therapy for tuberculosis. Int J Tuberc Lung Dis 2010;14:779-81.

17 AuYeung KY, DiCarlo L. Cost comparison of wirelessly vs directly observed therapy for adherence confirmation in anti-tuberculosis treatment. Int J Tuberc Lung Dis 2012;16:1498-1504. 